\title{
Comparison of an Additional Early Visit to Routine Postpartum Care on Initiation of Long- Acting Reversible Contraception: A Randomized Trial
}

Caitlin BERNARD, MD, $\mathrm{MSCl}^{1}$, Ms. Leping WAN, MPH ${ }^{2}$, Jeffrey F. PEIPERT, MD, PhD ${ }^{1}$, Tessa MADDEN, MD, $\mathrm{MPH}^{3}$

${ }^{1}$ Department of Obstetrics \& Gynecology, Indiana University School of Medicine, Indianapolis, Indiana

${ }^{2}$ Division of Clinical Research, Department of Obstetrics \& Gynecology, Washington University in St. Louis School of Medicine, St. Louis, Missouri

${ }^{3}$ Division of Family Planning, Department of Obstetrics \& Gynecology, Washington University in St. Louis School of Medicine, St. Louis, Missouri

\section{Address correspondence to:}

Caitlin Bernard, MD, MSCl, FACOG

Department of Obstetrics and Gynecology, Division of Family Planning

Indiana University School of Medicine

720 Eskenazi Ave FOB $3^{\text {rd }}$ Floor

Indianapolis, IN 46202

Email: caitlinbernardmd@gmail.com

Phone: 207-776-8255

Disclosures: Dr. Peipert receives research funding/support from Bayer, CooperSurgical/Teva, and Merck, and serves on advisory boards for CooperSurgical. Dr. Madden receives honorarium for serving on a data and safety monitoring committee for phase 4 studies of Bayer

Pharmaceuticals. Dr. Bernard \& Ms. Wan report no conflicts of interest.

Funding: This work was supported through a grant from the Society of Family Planning Research Fund. This publication was supported by the Clinical and Translational Science Award (CTSA) program of the National Center for Advancing Translational Sciences (NCATS) of the National Institutes of Health (NIH) under Award Numbers UL1 TR000448 and TL1 TR000449 and NIH Reproductive Epidemiology Training Grant number T32HD055172. The content is solely the responsibility of the authors and does not necessarily represent the official views of the NIH.

Short title: Early Postpartum Visit for LARC Initiation

Acknowledgements: Thank you to Michele Curran and Melissa Chapnick, without whom this project would not have been possible.

\section{Word Count}

Abstract $=245$

Manuscript text $=2500$ 
Objective: To investigate whether an early 3-week postpartum visit in addition to the standard 6-week visit increases LARC initiation by 8 weeks postpartum compared to the routine 6-week visit alone.

Study Design: We enrolled pregnant and immediate postpartum women into a prospective randomized, non-blinded trial comparing a single 6-week postpartum visit (routine care) to two visits at 3 and 6 weeks postpartum (intervention), with initiation of contraception at the 3-week visit, if desired. All participants received structured contraceptive counseling. Participants completed surveys in-person at baseline and at the time of each postpartum visit. A sample size of 200 total participants was needed to detect a 2 -fold difference in LARC initiation (20\% vs. 40\%).

Results: Between May 2016 and March 2017, 200 participants enrolled; outcome data are available for 188 . The majority of LARC initiation occurred immediately postpartum ( $25 \%$ of the intervention arm and $27 \%$ of the routine care arm). By 8 weeks postpartum, $34 \%$ of participants in the intervention arm initiated LARC, compared to $41 \%$ in the routine care arm $(p=0.35)$. Overall contraceptive initiation by 8 weeks was $83 \%$ and $84 \%$ in the intervention and routine care arms, respectively $(p=0.79)$. There was no difference between the arms in the proportion of women who attended at least one postpartum visit ( $70 \%$ vs. $74 \%, p=0.56)$.

Conclusion: The addition of a 3-week postpartum visit to routine care does not increase LARC initiation by 8 weeks postpartum. The majority of LARC users desired immediate rather than interval postpartum initiation.

Clinical Trial Registration: Clinicaltrials.gov NCT02769676 
Implications: The addition of a 3-week postpartum visit to routine care does not increase LARC or overall contraceptive initiation by 8 weeks post-partum when the option of immediate postpartum placement is available. The majority of LARC users desired immediate rather than interval postpartum initiation. 


\section{Introduction}

Most women return to sexual activity and ovulation before the 6-week postpartum visit(1). Therefore, it is recommended that women not exclusively breastfeeding initiate contraception by 3 weeks postpartum(1). However, only $20 \%$ of women use contraception in the month following delivery(2). Immediate postpartum (IPP) initiation of long acting reversible contraception (LARC) is a successful strategy $(3,4)$, but not all patients desire IPP initiation, there is a higher expulsion rate with IPP intrauterine device (IUD) placement(5), and access to IPP LARC is limited. Strategies for improving postpartum LARC access must address the multiple post-discharge barriers(6-11).

A common barrier to LARC initiation at a 6-week visit is difficulty excluding pregnancy(12), prompting a second visit, thus reducing the likelihood of initiating any method(13). LARC initiation before 4 weeks postpartum eliminates this concern, as pregnancy can always be ruled out(14). Additionally, many women lose public health insurance 6-8 weeks postpartum(15), making LARC unaffordable. Changing the model of postpartum care to include a 3-week visit with LARC initiation, if desired, has the potential to reduce these barriers.

Studies show 2-3 week postpartum IUD insertion is feasible and acceptable with lower expulsion rates compared to reported rates after IPP insertion(16-18). However, variable methodology and populations and lack of availability of IPP insertion leave unanswered questions. One option is to schedule one early visit, but this may miss an opportunity to address issues that arise later. Adding an early visit to routine care is one strategy that addresses barriers to LARC access and provides comprehensive care. We hypothesized that 
two planned visits, at 3- and 6-weeks postpartum, compared to one 6-week visit, would increase LARC initiation by 8 weeks postpartum.

\section{Materials and Methods}

This study was conducted from May 2016 to March 2017 at a tertiary academic medical center in St. Louis, Missouri. We obtained Institutional Review Board approval and registered at ClinicalTrials.gov (NCT02769676) prior to recruitment. We followed the Consolidated Standards of Reporting Trials (CONSORT) guidelines for reporting randomized trials.

We performed a parallel, randomized, non-blinded trial in which participants were randomized with 1:1 allocation to two arms: the routine care arm participants were scheduled for the routine 6-week postpartum visit, while the intervention arm participants were scheduled for visits at 3-and 6-weeks postpartum. We initially recruited from the inpatient postpartum service, but due to slow enrollment we additionally recruited antepartum women in the outpatient obstetrics clinic. Research assistants screened potential participants during postpartum hospitalization or at routine antepartum visits and willing participants underwent written informed consent. We included women aged $14-45, \geq 36$ weeks' gestation or postpartum, planning to deliver or delivered at our hospital, and planning to attend postpartum care at the outpatient clinic. We excluded women who were unable to be approached, incarcerated or non-English speaking. Participants recruited postpartum were excluded if they already received IPP LARC or sterilization or if they experienced abortion, stillbirth, or neonatal death in this pregnancy. 
After enrollment, all participants received contraceptive counseling based on the Contraceptive CHOICE Project model,(19) including information about all methods in order of effectiveness. Participants were block randomized in blocks of six and allocation was revealed using sequentially numbered sealed opaque envelopes. Participants in the routine care arm were scheduled for a 6-week postpartum visit, and a post-operative visit at 2-3 weeks for those who underwent cesarean delivery, per our institution's practice. The post-operative visit did not routinely include contraceptive initiation, but may at the discretion of the provider. Participants in the intervention arm were scheduled for visits at 3- and 6-weeks postpartum. For those who underwent cesarean delivery, the post-operative and 3-week visits were combined. The 3-week visit in the intervention arm included initiation of contraception, including LARC, if desired, along with standard postpartum care. Early visits were scheduled with residents, who inserted LARC under the supervision of generalists or family planning providers. Ultrasound-guidance was available, but never used. The 6-week visits were scheduled with nurse practitioners or residents. Participants received appointment reminders via phone, text, or email, depending on preference.

Our primary outcome was LARC initiation by 8 weeks postpartum. Secondary outcomes were overall contraceptive initiation by 8 weeks postpartum and postpartum visit attendance. Baseline and outcome data were determined through electronic medical record (EMR) review and participant report, which was obtained via in-person questionnaire at each visit or by telephone within 1 month of an unattended visit. No discrepancies were noted between EMR and participant report. Data collected included contraceptive use, initiation timing, and visit attendance. Participants who discontinued or switched methods were classified based on final 
method by 8 weeks. For participants who did not attend the 6 -week visit and were unreachable, we assumed their method was the most recently used method and included them in primary analysis based on allocated group. Participants who attended visits at the wrong timing were analyzed based on their allocated group. Participants for whom we had no contact after enrollment were excluded from the analysis. Participants received gift cards as follows: $\$ 20$ at enrollment, \$20 for attending the additional visit, and \$10 for each survey.

Data collection/management was performed using REDCap electronic data tools. Analyses were performed using SAS (Version 9.4, SAS Institute Inc., Cary, NC). All tests were two-sided with $p$-value $<0.05$ deemed statistically significant. Baseline characteristics were compared between the two arms using Student's t-test, Chi-square or Fisher's exact test, as appropriate. Primary and secondary outcomes were compared between the two arms using Chi-square test.

Using billing data, we estimated an outpatient postpartum LARC initiation rate of $20 \%$. We proposed that a 2 -fold increase to $40 \%$ would be clinically significant. Using $80 \%$ power to detect this difference, given an alpha (type 1) error of 0.05 , and accounting for $20 \%$ loss to follow-up, we estimated a sample size of 100 per arm.

Prior to study initiation, there was no insurance coverage for IPP LARC and it was rarely provided. After study initiation, Missouri State Medicaid authorized reimbursement for IPP LARC, and this practice was rapidly implemented. In order to evaluate the isolated effect of the post-discharge intervention, we performed post hoc analyses excluding participants who received IPP LARC or sterilization. Relative risks and 95\% confidence intervals were generated 
to estimate the effects of assigned arm and attendance to an early postpartum visit, respectively, on LARC and overall contraceptive initiation.

\section{Results}

A total of 200 patients were enrolled and randomized, 99 in the intervention arm and 101 in the routine care arm (Figure 1). Three were excluded post-randomization due to ineligibility: two enrolled postpartum in error (one already received an IPP IUD and one did not plan to attend our clinic) and one enrolled antepartum but underwent hysterectomy. Four participants in the intervention and five in the routine care arm were lost to follow-up, leaving 188 for analysis (93 in the intervention, 95 in the routine care arm). The two arms were similar (Table 1), including predominantly young, single, multiparous, black, low-income women, using Medicaid insurance, with a high rate of unintended pregnancy, consistent with our urban hospital-based clinic population. In each arm, approximately $1 / 3$ of participants enrolled antepartum and $2 / 3$ enrolled postpartum. At enrollment, $94 \%$ desired postpartum contraception; $77 \%$ preferred initiation as soon as possible, while $15 \%$ preferred initiation at the 6 -week visit. After contraceptive counseling, approximately $40 \%$ in each arm planned to initiate LARC (see Table 1 for method breakdown). On enrollment, when queried about preference for postpartum visit timing, 62\% preferred within 4 weeks, $28 \%$ preferred in 6 weeks, and only $2 \%$ preferred multiple visits. Preference for a visit within 4 weeks did not differ based on parity (multiparous $58 \%$ vs. nulliparous $73 \%, p=0.086$ ), but differed slightly based on enrollment timing (antepartum 52\% vs. postpartum 67\%, p=0.047). 
The two arms were similar in rate of LARC initiation by 8 weeks postpartum (34\% [32/93] in the intervention vs. $41 \%$ [39/95] in the routine care arm; $p=0.35$ ) (Table 2). Most LARC users chose IPP initiation (25\% [23/93] of the intervention arm [8 IUDs and 15 implants] and $27 \%$ [26/95] of the routine care arm arm [7 IUDs and 19 implants]). In the intervention arm, 4\% (4/93) initiated LARC at the 3-week visit (3 IUDs and one implant) and 5\% (5/93) initiated LARC at the 6-week visit (4 IUDs and one implant). In the routine care arm, 3\% (3/95) initiated LARC at an early post-operative visit (4 implants) and 11\% (10/95) initiated LARC at the 6-week visit (4 IUDs and 6 implants). One participant in the intervention arm and 3 in the routine care arm received injectable contraception IPP, then initiated LARC between 5-7 weeks postpartum. IUD expulsions were rare; only one participant in the routine care arm who received immediate post-cesarean IUD insertion experienced a partial expulsion 10 days postpartum. Overall contraceptive initiation by 8 weeks also was similar between the two arms $(83 \%$ [77/93] in the intervention vs. $84 \%$ [80/95] in the routine care arm; $p=0.79)$. Most reversible non-LARC users also initiated use prior to hospital discharge (24\% [22/93] of the intervention and 25\% [24/95] of the routine care arm).

Only $46 \%$ [43/93] of the intervention arm attended the 3-week postpartum visit. The two arms were similar in attendance to at least one visit (70\% [65/93] in the intervention vs. $74 \%$ [70/95] in the routine care arm, $p=0.56)$ and attendance to the 6 -week visit $(54 \%$ [50/93] in the intervention vs. $65 \%$ [62/95] in the routine care arm, $p=0.11$ ) (Table 3). Only 30\% [28/93] of the intervention arm and $17 \%$ [16/95] of the routine care arm attended two visits. Receiving IPP LARC or sterilization did not impact postpartum visit attendance (66\% [38/58] of women who received IPP LARC or sterilization attended at least one visit vs. 75\% [98/130] of women who 
did not, $\mathrm{p}=0.16)$. Approximately $90 \%$ of women who attended a visit reported somewhat or complete visit satisfaction, with similar ratings for each arm and for both early and routine postpartum visits.

To evaluate the effect of the post-discharge intervention we performed post hoc analyses excluding participants who received IPP LARC or sterilization. This cohort includes 130 participants, 66 in the intervention and 64 in the routine care arm. There was no difference in LARC initiation (14\% [9/66] in the intervention vs. $20 \%[13 / 64]$ in the routine care arm, $p=0.31)$ or overall contraceptive initiation (76\% [50/66] in the intervention vs. 77\% [49/64] in the routine care arm, $p=0.91$ ). Within this cohort we also explored whether attendance to an early visit impacted LARC or overall contraceptive initiation; 36\% (47/130) attended an early visit and $64 \%(83 / 130)$ did not. There was no difference in LARC initiation (21\% [10/47] of participants who attended an early visit vs. $14 \%[12 / 83]$ of those who did not; $p=0.32)$. However, participants who attended an early visit were more likely to initiate any contraceptive method by both 4 weeks (66\% [31/47] vs. 41\% [34/83]; RR 1.61, 95\% Cl 1.16-2.24) and 8 weeks postpartum (87\% [41/47] vs. 70\% [58/83]; RR 1.25, 95\% Cl 1.04-1.49).

Because $27 \%$ of the routine care arm also attended an early postoperative visit after cesarean delivery, we performed a post hoc analysis excluding participants who underwent cesarean. There was still no difference in initiation of LARC (31\% [20/65] vs. 38\% [23/60], $\mathrm{p}=0.37)$ or any contraception $(77 \%[50 / 65]$ vs. $80 \%[48 / 60], p=0.68)$ or attendance to at least one postpartum visit (65\% [42/65] vs. $67 \%$ [40/60], $p=0.81)$.

\section{Discussion}


We found that planned 3- and 6-week postpartum visits compared to a single, routine 6week visit did not increase LARC or overall contraceptive initiation rates or attendance to at least one postpartum visit. Among the participants who did not receive IPP LARC or sterilization, there was still no difference in LARC or overall contraceptive initiation between the two groups. However, participants who attended an early postpartum visit were more likely to initiate contraception by both 4 and 8 weeks postpartum, compared to those who did not attend an early visit. The majority of LARC and overall contraceptive initiation occurred immediately postpartum and we had high rates of contraceptive use by 8 weeks postpartum (>80\% for each group).

Recent studies have evaluated the feasibility, safety and acceptability of early postpartum IUD insertion $(16,17)$. Zerden et al. found that IUD insertion at 2-3 weeks postpartum had high patient acceptability and method continuation rates and a lower expulsion rate compared with published reports of IPP insertion(16). Baldwin, et al. randomized women to 3 or 6 -week postpartum IUD insertion and found similarly high IUD use (75\% vs. $80 \%$ ) at 3 months postpartum in both groups (17). Together with our findings of high uptake of IPP contraception, this evidence indicates that the timing of initiation may not be as important as the availability of multiple opportunities for postpartum LARC services.

Despite most women expressing a preference for a visit within 4 weeks, only $46 \%$ of the intervention group attended the 3-week visit. However, our findings suggest that attending an earlier visit may improve overall contraceptive initiation compared to attending only a later postpartum visit. The results from Baldwin, et al. support this, as they found that women 
randomized to 3-week insertion were more likely to receive an IUD by 45 days postpartum and prior to resuming intercourse,(17) indicating that planning for earlier initiation may help circumvent barriers. Multiple socioeconomic and cultural factors may influence ability to attend a postpartum visit and interventions to improve postpartum contraceptive use may have limited success in women who have difficulty attending visits (20-22). This reinforces the importance of the availability of IPP LARC, and indicates the need to individualize postpartum care, including visit timing.

The strengths of this study are the randomized trial design, implementation within a real-world setting, and minimal loss to follow-up. The main limitation was the unexpected implementation of IPP LARC during our study, resulting in high rates of IPP LARC initiation. Our intervention was designed to provide the most benefit for women discharged without an effective method, and we suspect that the high rate of IPP LARC uptake mitigated the potential benefit of an early postpartum visit. Therefore the results of this study may not be generalizable to populations without access to IPP LARC. The availability of IPP LARC increased LARC use above the estimated $20 \%$ baseline. Additionally, $27 \%$ of participants in the routine care arm attended an early post-operative visit following cesarean delivery and some participants initiated contraception at this visit, making the routine care arm more similar to the intervention. These factors may have biased our study towards showing no difference between the two arms. Study participation may have increased LARC use due to the additional counseling and postpartum visit attendance due to receiving appointment reminders, neither of which are institutional standard of care. 
There has been increasing appreciation for the needs of postpartum women, (23) but we need to continue to optimize their care, including contraceptive initiation. Our study indicates that when available, most women prefer IPP contraceptive initiation. For those who don't, planning for an early postpartum visit may not be sufficient, but attending an early visit may be beneficial for improving contraceptive initiation. Further research should evaluate how to improve access to highly effective contraception for women across the postpartum phase, including the early postpartum period. 


\section{References}

1. Speroff L, Mishell DR, Jr. The postpartum visit: it's time for a change in order to optimally initiate contraception. Contraception. 2008;78(2):90-8.

2. Conde-Agudelo A, Rosas-Bermudez A, Kafury-Goeta AC. Birth spacing and risk of adverse perinatal outcomes: a meta-analysis. JAMA. 2006;295(15):1809-23.

3. Sothornwit J, Werawatakul Y, Kaewrudee S, Lumbiganon P, Laopaiboon M. Immediate versus delayed postpartum insertion of contraceptive implant for contraception. Cochrane Database Syst Rev. 2017;4:CD011913.

4. Lester F, Kakaire O, Byamugisha J, Averbach S, Fortin J, Maurer R, et al. Intracesarean insertion of the Copper T380A versus 6 weeks postcesarean: a randomized clinical trial. Contraception. 2015;91(3):198-203.

5. Lopez LM, Bernholc A, Hubacher D, Stuart G, Van Vliet HA. Immediate postpartum insertion of intrauterine device for contraception. Cochrane Database Syst Rev. 2015(6):CD003036.

6. Lopez LM, Grey TW, Chen M, Hiller JE. Strategies for improving postpartum contraceptive use: evidence from non-randomized studies. Cochrane Database Syst Rev. 2014(11):CD011298.

7. Lopez LM, Hiller JE, Grimes DA, Chen M. Education for contraceptive use by women after childbirth. Cochrane Database Syst Rev. 2012(8):CD001863.

8. Dahlke JD, Terpstra ER, Ramseyer AM, Busch JM, Rieg T, Magann EF. Postpartum insertion of levonorgestrel--intrauterine system at three time periods: a prospective randomized pilot study. Contraception. 2011;84(3):244-8.

9. Whitaker AK, Endres LK, Mistretta SQ, Gilliam ML. Postplacental insertion of the levonorgestrel intrauterine device after cesarean delivery vs. delayed insertion: a randomized controlled trial. Contraception. 2014;89(6):534-9.

10. Chen BA, Reeves MF, Hayes JL, Hohmann HL, Perriera LK, Creinin MD. Postplacental or delayed insertion of the levonorgestrel intrauterine device after vaginal delivery: a randomized controlled trial. Obstet Gynecol. 2010;116(5):1079-87.

11. Zerden ML, Tang JH, Stuart GS, Norton DR, Verbiest SB, Brody S. Barriers to Receiving Longacting Reversible Contraception in the Postpartum Period. Womens Health Issues. 2015;25(6):616-21.

12. Ogburn JA, Espey E, Stonehocker J. Barriers to intrauterine device insertion in postpartum women. Contraception. 2005;72(6):426-9.

13. Bergin A, Tristan S, Terplan M, Gilliam ML, Whitaker AK. A missed opportunity for care: two-visit IUD insertion protocols inhibit placement. Contraception. 2012;86(6):694-7.

14. Curtis KM, Jatlaoui TC, Tepper NK, Zapata LB, Horton LG, Jamieson DJ, et al. U.S. Selected Practice Recommendations for Contraceptive Use, 2016. MMWR Recomm Rep. 2016;65(4):1-66. 15. Walker LO, Wilging S. Rediscovering the "M" in "MCH": maternal health promotion after childbirth. J Obstet Gynecol Neonatal Nurs. 2000;29(3):229-36.

16. Zerden ML, Stuart GS, Charm S, Bryant A, Garrett J, Morse J. Two-week postpartum intrauterine contraception insertion: a study of feasibility, patient acceptability and short-term outcomes. Contraception. 2017;95(1):65-70.

17. Baldwin MK, Edelman AB, Lim JY, Nichols MD, Bednarek PH, Jensen JT. Intrauterine device placement at 3 versus 6 weeks postpartum: a randomized trial. Contraception. 2016;93(4):356-63.

18. Chen MJ, Hou MY, Hsia JK, Cansino CD, Melo J, Creinin MD. Long-Acting Reversible Contraception Initiation With a 2- to 3-Week Compared With a 6-Week Postpartum Visit. Obstet Gynecol. 2017;130(4):788-94.

19. Madden T, Mullersman JL, Omvig KJ, Secura GM, Peipert JF. Structured contraceptive counseling provided by the Contraceptive CHOICE Project. Contraception. 2013;88(2):243-9. 
20. Bryant AS, Haas JS, McElrath TF, McCormick MC. Predictors of compliance with the postpartum visit among women living in healthy start project areas. Matern Child Health J. 2006;10(6):511-6.

21. Wilcox A, Levi EE, Garrett JM. Predictors of Non-Attendance to the Postpartum Follow-up Visit. Matern Child Health J. 2016;20(Suppl 1):22-7.

22. Simmons KB, Edelman AB, Li H, Yanit KE, Jensen JT. Personalized contraceptive assistance and uptake of long-acting, reversible contraceptives by postpartum women: a randomized, controlled trial. Contraception. 2013;88(1):45-51.

23. Tully KP, Stuebe AM, Verbiest SB. The fourth trimester: a critical transition period with unmet maternal health needs. Am J Obstet Gynecol. 2017. 
Table 1: Comparison of participant baseline characteristics by study arm: intervention ( 3 and 6 week postpartum visits) versus routine care shows no differences between the two arms.

\begin{tabular}{|c|c|c|}
\hline Characteristic & $\begin{array}{l}\text { Intervention Arm } \\
\qquad(n=93)\end{array}$ & $\begin{array}{l}\text { Routine Care Arm } \\
\qquad(n=95)\end{array}$ \\
\hline Age, in years & $26.0 \pm 5$ & $25.3 \pm 5$ \\
\hline \multicolumn{3}{|l|}{ Race } \\
\hline Black & $84(90.3)$ & $84(88.4)$ \\
\hline White & $5(5.4)$ & $10(10.5)$ \\
\hline Other & $4(4.3)$ & $1(1.1)$ \\
\hline \multicolumn{3}{|l|}{ Ethnicity } \\
\hline Non-Latina & $84(90.3)$ & $88(92.6)$ \\
\hline Latina & $9(9.7)$ & $7(7.4)$ \\
\hline \multicolumn{3}{|l|}{ Relationship status } \\
\hline Single/never married & $51(54.8)$ & $56(58.9)$ \\
\hline Married/cohabitating & $38(41.0)$ & $37(38.9)$ \\
\hline Separate/divorced/widowed & $3(3.2)$ & $2(2.1)$ \\
\hline Missing & $1(1.1)$ & 0 \\
\hline Previous delivery & $74(79.6)$ & $72(75.8)$ \\
\hline \multicolumn{3}{|l|}{ Educational attainment } \\
\hline Less than high school & $16(17.2)$ & $12(12.6)$ \\
\hline High school/GED & $35(37.6)$ & $47(49.5)$ \\
\hline Some college/associates degree & $32(34.4)$ & $31(32.6)$ \\
\hline Vocational/technical school & $5(5.4)$ & $4(4.2)$ \\
\hline College degree & $5(5.4)$ & $1(1.1)$ \\
\hline \multicolumn{3}{|l|}{ Employment status } \\
\hline Working full-time & $28(30.1)$ & $33(34.7)$ \\
\hline Working part-time & $17(18.3)$ & $14(14.7)$ \\
\hline Unemployed & $32(34.4)$ & $30(31.6)$ \\
\hline Disabled/sick leave/other & $11(11.8)$ & $12(12.6)$ \\
\hline Full-time student & $5(5.4)$ & $6(6.3)$ \\
\hline Currently receiving public assistance & $81(87.1)$ & $86(90.5)$ \\
\hline Trouble paying for basic necessities & $31(33.3)$ & 36 (37.9) \\
\hline Missing & $2(2.2)$ & $3(3.2)$ \\
\hline \multicolumn{3}{|l|}{ Mode of delivery } \\
\hline Vaginal & $65(70.0)$ & $60(63.2)$ \\
\hline Cesarean & $28(30.1)$ & $35(36.8)$ \\
\hline Prior unintended pregnancy & $68(73.1)$ & $73(76.8)$ \\
\hline Current unintended pregnancy & $59(63.4)$ & $64(68.1)$ \\
\hline Missing & 0 & $1(1.1)$ \\
\hline \multicolumn{3}{|l|}{ Desires future pregnancy } \\
\hline No & $53(57.0)$ & $53(55.8)$ \\
\hline Yes & 25 (26.9) & $32(33.7)$ \\
\hline Unsure/Ambivalent & $15(16.1)$ & $10(10.5)$ \\
\hline \multicolumn{3}{|l|}{ Desired timing of future pregnancy } \\
\hline In $1-2$ years & $4(10.0)$ & $2(4.8)$ \\
\hline In $>2$ years & $33(82.5)$ & $35(83.3)$ \\
\hline Unsure & $3(7.5)$ & $4(9.5)$ \\
\hline Missing & 0 & $1(2.4)$ \\
\hline \multicolumn{3}{|c|}{ Planned postpartum contraceptive method* } \\
\hline LARC & $41(44.1)$ & $39(41.1)$ \\
\hline IUD & $15(16.1)$ & $14(14.7)$ \\
\hline Implant & $26(28.0)$ & $25(26.3)$ \\
\hline
\end{tabular}




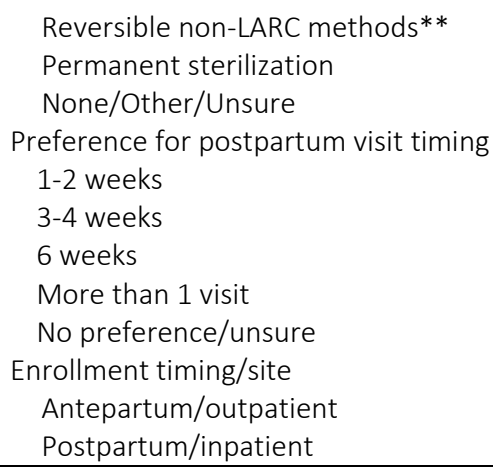

Values are expressed as mean \pm standard deviation for continuous variables or number of subjects (percentage) for categorical variables.

GED = General Education Development/Diploma

*Total $>100 \%$ due to multiple possible responses

LARC $=$ long-acting reversible contraception

IUD = intrauterine device

Implant = subdermal contraceptive implant

**Reversible non-LARC methods = Depo-medroxyprogesterone acetate (DMPA), oral contraceptive pills, contraceptive patch, or contraceptive ring. 
Table 2: Comparison of participant contraceptive method initiation by study arm: intervention ( 3 and 6 week postpartum visits) versus routine care.

\begin{tabular}{|c|c|c|c|}
\hline & $\begin{array}{l}\text { Intervention Arm } \\
\qquad(n=93)\end{array}$ & $\begin{array}{l}\text { Routine Care Arm } \\
\qquad(n=95)\end{array}$ & p-value** \\
\hline \multicolumn{4}{|l|}{ Immediate Postpartum } \\
\hline LARC & $23(24.7)$ & $26(27.4)$ & \\
\hline Intrauterine device & $8(8.6)$ & $7(7.4)$ & \\
\hline Implant & $15(16.1)$ & $19(20.0)$ & \\
\hline Reversible non-LARC* & $22(23.7)$ & $24(25.3)$ & \\
\hline Sterilization & $4(4.3)$ & $5(5.3)$ & \\
\hline Cumulative total use & $49(52.7)$ & $55(57.9)$ & 0.47 \\
\hline \multicolumn{4}{|l|}{ Discharge to $\leq 4$ Weeks Postpartum } \\
\hline LARC & $4(4.3)$ & $3(3.2)$ & \\
\hline Intrauterine device & $3(3.2)$ & & \\
\hline Implant & $1(1.1)$ & $4(4.2)$ & \\
\hline Reversible non-LARC* & $11(11.8)$ & $2(2.1)$ & \\
\hline Sterilization & 0 & 0 & \\
\hline Cumulative total use & $64(68.9)$ & $0(63.2)$ & 0.41 \\
\hline \multicolumn{4}{|l|}{$>4$ to 8 Weeks Postpartum } \\
\hline LARC & $5(5.4)$ & $10(10.5)$ & \\
\hline Intrauterine device & $4(4.3)$ & $4(4.2)$ & \\
\hline Implant & $1(1.1)$ & $6(6.3)$ & \\
\hline Reversible non-LARC* & $5(5.4)$ & $9(9.5)$ & \\
\hline Sterilization & $3(3.2)$ & $1(1.1)$ & \\
\hline LARC use by 8 weeks postpartum & $32(34.4)$ & $39(41.1)$ & 0.35 \\
\hline Any method use by 8 weeks postp & $77(82.8)$ & $80(84.2)$ & 0.79 \\
\hline
\end{tabular}

Values are expressed as number of subjects (percentage).

* Reversible non-LARC methods = Depo-medroxyprogesterone acetate (DMPA), oral contraceptive pills, contraceptive patch, or contraceptive ring.

**All p-values obtained by Chi-square testing 
Table 3: Comparison of postpartum visit attendance by study arm: intervention ( 3 and 6 week postpartum visits) versus routine care.

\begin{tabular}{|lccc|}
\hline & $\begin{array}{c}\text { Intervention Arm } \\
(n=93)\end{array}$ & $\begin{array}{c}\text { Routine Care Arm } \\
(n=95)\end{array}$ & p-value* \\
Early postpartum/postoperative visit & $43(46.2)$ & $26(27.4)$ & 0.007 \\
6-week postpartum visit & $50(53.7)$ & $62(65.3)$ & 0.11 \\
Two visits & $28(30.1)$ & $16(16.8)$ & 0.03 \\
At least one visit & $65(69.9)$ & $70(73.7)$ & 0.56 \\
\hline
\end{tabular}

Values are expressed as number of subjects (percentage).

*All p-values obtained by Chi-square testing 


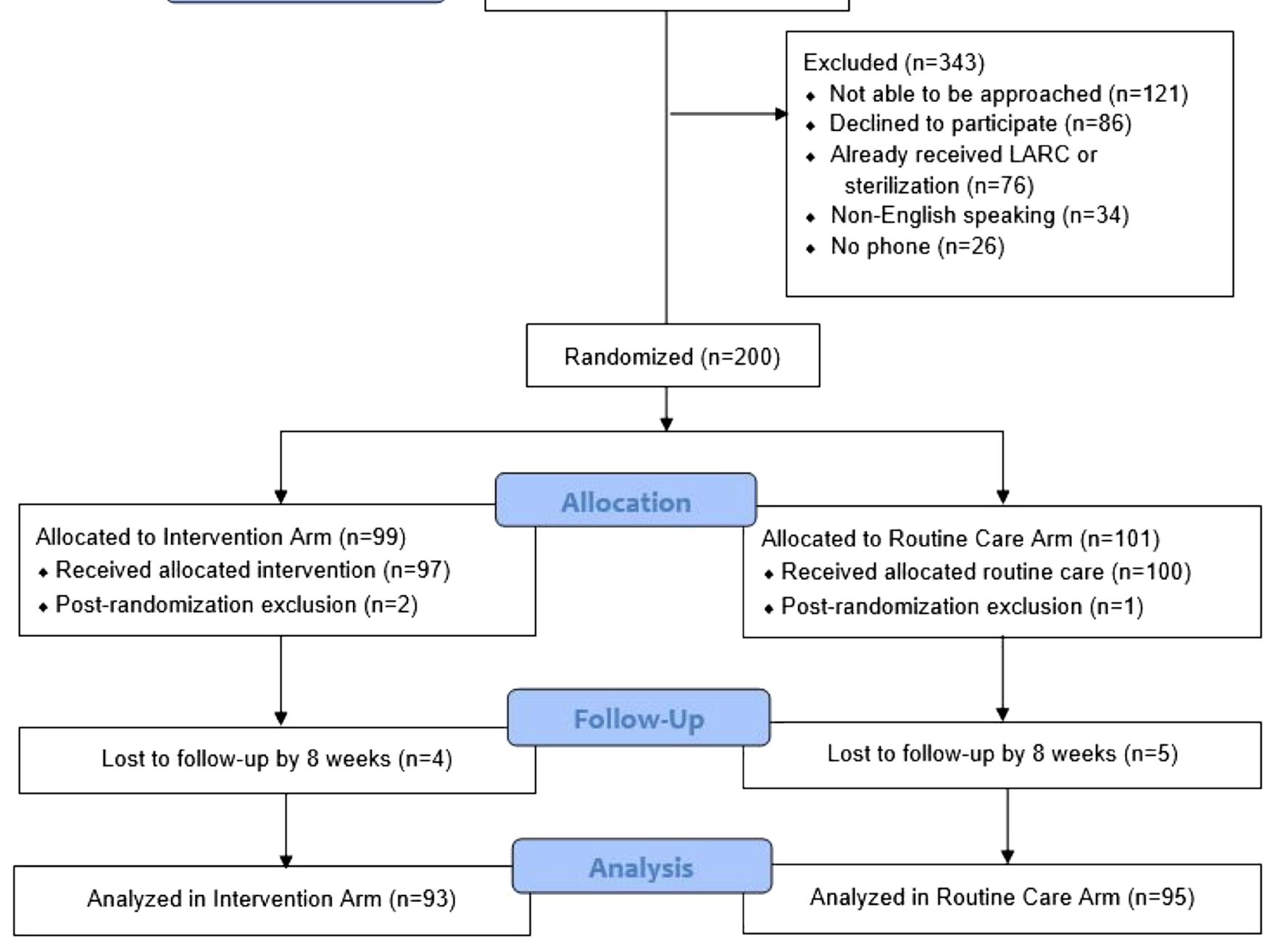

Figure 1 